\section{g) Photographie.}

D.R.P. 549380 vom 7. Juni 1930. I. G. Farbenindustrie A. G., Frankfurt a. M., und Technicolor Motion Picturen Corporation, Boston (U.S.A.).

Verfahren zum Härten der Gelatineschicht von für das Einsaugedruckverfahren (Imbibitionsdruck) bestimmtem Blankfilm, dadurch gekennzeichnet, daß die Gelatineschicht ohne Zusatz eines Härtungsmittels gegossen und nach dem Erstarren, aber vor dem Trocknen mit einem Härtungsmittel behandelt wird.

\section{i) Verschiedenes.}

D.R.P. 545242 vom 27. Mai 1922. The New Jersey Zinc Company, New York.

1. Verfahren zum Herstellen von Zinkoxyd hohen Feinheitsgrades durch Einführen von Zinkdampf in eine bewegte oxydierende Atmosphäre, dadurch gekennzeichnet, daß der auf etwa $2000^{\circ} \mathrm{C}$ und höher überhitzte Zinkdampf mit hoher Geschwindigkeit in Form eines Strahles durch eine Düse in die oxydierende Atmosphäre eingeblasen wird.

3. Vorrichtung nach Anspruch 2, dadurch gekennzeichnet, daß das Destillationsgefäß mit Elektroden ausgerüstet ist, von denen die eine gleichzeitig als Austrittsdüse dient.

D.R.P. 545094 vom 28. April 1928. I. G. Farbenindustrie A. G., Frankfurt a. M.

Verfahren zur Herstellung von Emulgiermitteln, dadurch gekennzeichnet, daß man andere Oxydationsprodukte von Paraffinkohlenwasserstoffen, Wachsen u. dgl. als Ozonide oder die daraus isolierten sauren Bestandteile für sich oder in Mischung miteinander oder in Mischung mit anderen Stoffen unter solchen Bedingungen chloriert, daß die Bildung von Harzen vermieden wird, d. h. zweckmäßig unter Verwendung eines geeigneten Lösungsmittels bei Temperaturen unter $100^{\circ}$ mit Chlormengen bis $z u$ etwa 40 Proz. der verwendeten Ausgangsmaterialien, und die dabei entstandenen Chlorierungsprodukte zwecks Neutralisation oder zwecks Ersatzes der Chloratome durch Hydroxylgruppen und gleichzeitiger Neutralisation mit basischen Mitteln behandelt.

D.R.P. 545339 vom 19. Februar 1930. Dr. Alexander Wacker Gesellschaft für elektrochemische Industrie G. m. b. H., München.

Verfahren zur Herstellung verstäubbarer Pflanzenschutzmittel, enthaltend wasserabstoßende Zusatzstoffe, dadurch gekennzeichnet, daß den wirksamen Anteilen durch wasserlösliche hydrophile Zusätze Wasserbenetzbarkeit erteilt bzw. vorhandene Benetzbarkeit verstärkt wird.

D.R.P. 545193 vom 13. Juli 1930. Metallgesellschaft A. G., Frankfurt a. $M$.

Verfahren zum Wiederaustreiben von Gasen, Dämpten, Gasdampfgemischen od. dgl., insbesondere von höher siedenden Gasen oder Dämpfen, z. B. von Solventnaphtha, aus großoberflächigen Körpern, wie z. B. Aktivkohle, Silikagel od. dgl., unter Verwendung von Spüldampf, dadurch gekennzeichnet, daß während der Spülperiode im Adsorber bei höheren Drucken, als sie durch die Widerstände der Leitungen und den Gegendruck der Entlüftungsleitung des
Kondensators an sich gegeben sind, gearbeitet wird, $z$. B. dadurch, daß man in die Destillationsaustrittsleitungen bzw. in die Apparate der Entlüftungsleitungen hinter den Adsorber einen besonderen Strömungswiderstand, wie z. B. ein Reduzierventil, Drosselscheibe, Düse od. dgl. einbaut.

D.R.P. 545264 vom 19. September 1930. I. G. Farbenindustrie A. G., Frankfurt a. M.

Verfahren zur Herstellung emulgierbarer Fettgemische, darin bestehend, daß ungesättigte Fettsäuren bzw. ihre Ester mit schwefligsauren Salzen unter gleichzeitiger Einwirkung von Oxydationsmitteln mit oder ohne Zusatz von Katalysatoren unvollständig sulfitiert werden.

\section{k) Kautschuk und Klebemitte}

D.R.P. 543153 vom 22. Juli 1927. The Anode Rubber Company Limited, Guernsey (England).

Verfahren zur Herstellung von Kautschukwaren mit angelagerten oder eingebetteten Textileinlagen.

Verfahren zur Verbindung einer Faserstofflage mit Niederschlägen, die aus koagulierbaren wässerigen Dispersionen kautschukartiger Stoffe mittels durch die Unterlage hindurch stattfindender Ionenwanderung erzeugt werden, dadurch gekennzeichnet, daß die Faserstofflage vor der Bildung des Niederschlages mit der gleichen oder einer verschiedenen Dispersion des $\mathrm{zu}$ agglomerierenden Stoffes vollkommen durchtränkt wird.

D.R.P. 544879 vom 9. November 1927. Ernst Buhtz, Berlin.

Verfahren zur Herstellung eines stärkehaltigen Kleisters durch Aufbringen von Stärkẹsuspensionen auf geheizte Flächen, gegebenenfalls in Anwesenheit nicht neutral reagierender flüchtiger Mittel, dadurch gekennzeichnet, daß den Stärkesuspensionen Hefe zugesetzt wird.

D.R.P. 544641 vom 16. September 1928. The Anode Rubber Company (England) Limited, London.

Verfahren zur Entwässerung von Schichten, die aus wässerigen Kautschukdispersionen oder ähnlichen koagulierbaren Stoffen erhalten werden, dadurch gekennzeichnet, daß um die auf einer gekrümmten, starren Unterlage zu entwässernde Schicht ein biegsames, wasserdurchlässiges Entwässerungsblatt derart unter Spannung herumgeführt wird, daß der längs einer Erzeugenden der krummen Fläche auftretende Entwässerungsdruck sich allmählich auf die ganze Oberfläche der Schicht fortpflanzt bzw. ausdehnt.

\section{Berichtigung.}

In der Arbeit von R. v. Dallwitz-Waegner, Oberflächenspannung und molekularer Aufbau der Flüssigkeiten und festen Körper im Sinne der ,,Raumenergetik" in Kolloid-Z. 70, H. 1, muß auf S. 40, Gleichung (3) der Wert von $L$ noch mit $\sqrt{Z_{\mathrm{ccm}}}$ $=0,7746.1 / \mathrm{\gamma} / \mathrm{M}$ multipliziert werden, wenn $Z_{\mathrm{ccm}}$ die Molekelanzahl der Stoffe je ccm Rauminhalt ist. Die ,Ladung" L ist dann ein spezifisches Kennzeichen bei "Stoffmischungen und Lösungen usw. In meiner Hauptarbeit , Raumenergetik“, die bei Vieweg \& Sohn erscheint, ist" das näher ausgeführt. 